\title{
Modelling temperature variability in batch retorts and its impact on lethality distribution
}

\author{
Szabolcs Varga ${ }^{a}$, Jorge C. Oliveira ${ }^{b, *}$, Chantal Smout ${ }^{c}$, Mark E. Hendrickx ${ }^{\mathrm{c}}$ \\ a Escola Superior de Biotecnologia, Rua Dr António Bernardino de Almeida, 4200 Porto, Portugal \\ $\mathrm{b}$ Department of Food Science and Technology, University College Cork, Cork, Ireland \\ c Faculty of Agriculture and Applied Biological Sciences, Catholic University of Leuven, Kardinaal Mercierlaan 92, 3001 Heverlee, Belgium
}

Keywords: Conduction-heating; Mathematical modelling; Finite elements; In-pack sterilisation; Time-series

\begin{abstract}
Experimental time-temperature distributions from two different industrial scale retort systems were statistically analysed. The retort temperature was modelled as the sum of a trend value and a residual, with the trend temperatures being simple functions of time. The residuals were modelled using time-series. The resulting impact on the lethality distribution was assessed by calculating the $F$-value distribution in the centre of cans simulated via a conduction-heating finite element model for 180 simulated temperature histories. Comparing the distributions obtained with those calculated using the actual experimental temperature histories validated the applicability of this approach. The results indicated that the experimental and the modelled average lethalities were statistically similar at $95 \%$ confidence. The standard deviation was also similar for the $F$-value up to the end of holding but larger for the modelled distribution when considering the whole cycle, which was attributed to a correlation between the heating and cooling parameters that was not considered in the model. (c) 2000 Published by Elsevier Science Ltd. All rights reserved.
\end{abstract}

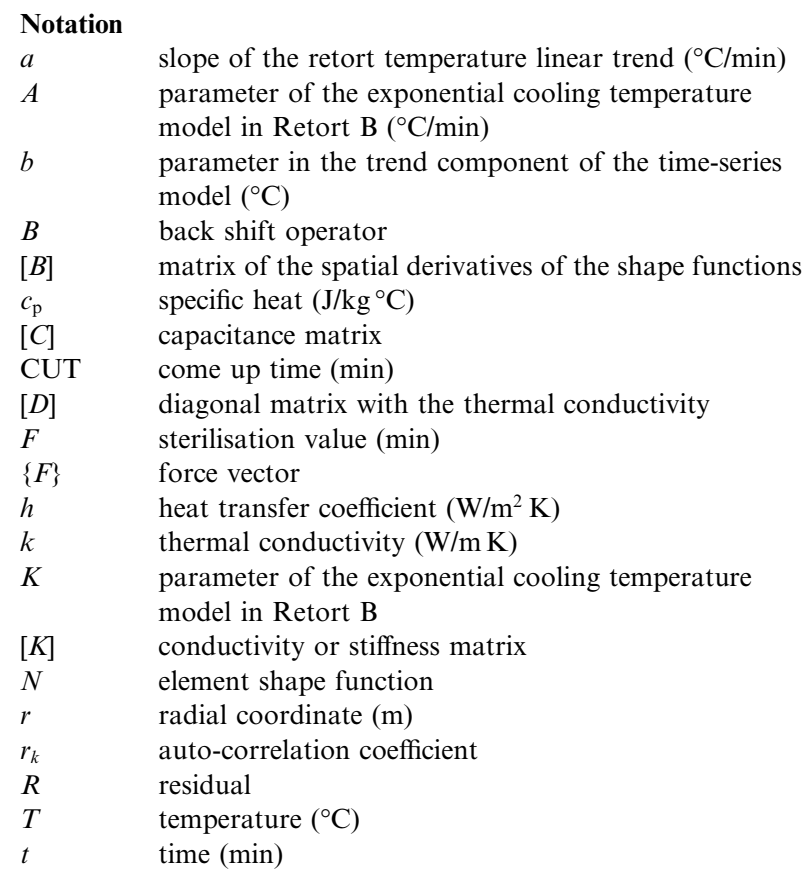

${ }^{*}$ Corresponding author. Fax: +353-21-276398.
E-mail address: j.oliveira@ucc.ie (J.C. Oliveira).

$\begin{array}{ll}V & \text { volume }\left(\mathrm{m}^{3}\right) \\ W_{i} & \text { weighing function } \\ y & \text { response variable } \\ z & \begin{array}{l}\text { axial coordinate (in heat transfer equations), } m \text { or } \\ \text {-value of the target micro-organism (lethality } \\ \text { equations) }\left({ }^{\circ} \mathrm{C}\right)\end{array} \\ & \text { thermal diffusivity }\left(\mathrm{m}^{2} / \mathrm{s}\right) \\ \alpha & \text { coefficient in the time-series model } \\ \phi & \text { coefficient in the time-series model } \\ \theta & \text { density }\left(\mathrm{kg} / \mathrm{m}^{3}\right) \\ \rho & \\ \text { Subscripts } & \\ 0 & \text { initial } \\ \text { ecu } & \text { end of come up } \\ \mathrm{H} & \text { holding } \\ \text { heat } & \text { heating (come-up }+ \text { holding) } \\ \mathrm{n} & \text { nodal } \\ \mathrm{R} & \text { retort } \\ \text { tot } & \text { total process } \\ \text { Superscripts } & \\ \mathrm{e} & \text { element } \\ \mathrm{T} & \text { transpose }\end{array}$

\section{Introduction}

Conventional temperature distribution tests are performed with a view to identify the coldest spot in the 
retort in order to specify the processing requirements. However, from a statistical point of view it would be preferable to describe the process variability according to probabilities and frequency distributions for which it would be necessary to consider a large number of data and factors.

Some factors vary only in space (such as headspace volume and initial temperature), others in space and time (such as retort temperature and surface heat transfer coefficient) "in a more or less noisy and unpredictable way" (Nicolai \& Baerdemaeker, 1992). There are a number of available published data on the statistical analysis of thermal processes related to factors varying in the space domain (e.g., Hicks, 1961; Lund, 1978; Patino \& Heil, 1985; Hayakawa, Massaguer \& Trout, 1988; Nicolai, 1994), however the available information on the effect of factors that vary also in time is quite limited.

A straightforward method to analyse retort temperature and heat transfer coefficient distributions would be an experimental approach (Tung, Britt \& Ramaswamy, 1990; Adams \& Hardt-English, 1990; Park, Cables \& Collins, 1990; Campbell \& Ramaswamy, 1992). However, the statistical reliability of the experimental retort heat distribution tests is highly limited by their time consumption and cost. In a typical study only one experimental run is made with $20-30$ thermocouples distributed in a fully loaded retort and the temperature is recorded to locate the coldest spot in the equipment (Tung et al., 1990), which is usually identified as the location of the thermocouple that resulted in the lowest average temperature during the holding phase of the sterilisation cycle. The information provided by such tests is not satisfactory due to several reasons such as the 'real' cold point might not be monitored, the lowest average holding temperature may not result in the lowest lethality and batch to batch variability can change its location. Also, important sources of variability are overlooked by not considering the temperature data from the come-up, come-down and cooling phases.

The use of powerful numerical methods can contribute to a wider body of knowledge, by allowing comprehensive analysis with a large amount of data in an inexpensive manner. In order to perform a reliable heat distribution test via mathematical simulations, three conditions must be fulfilled simultaneously: (i) adequate heat penetration model, (ii) appropriate statistical method to 're-generate' the reality and particularly its variability and in relation to that (iii) accurate estimates of the magnitude of the uncertainty.

The general objective of this work was to develop a modelling approach using an adequate statistical method able to mimic closely retort temperature variability in terms of its actual impact on lethality distribution. Experimental data are required to estimate model parameters, which therefore have a direct physical meaning, so that a proper link between experimental data and model results can be made. Once a time-series model has been extracted from experimental temperature distributions, a large number of temperature histories can be simulated and an $F$-value distribution can be properly inferred. Such model also has the advantage of providing a quantifiable and concise way of describing temperature variability, allowing for an objective comparison between different retorts and/or cycles.

\section{Materials and methods}

\subsection{General concept}

The process analysis is made by determining the $F$ value in the geometrical centre of a can subjected to the bulk (retort) temperature in question, considering that (i) there is a finite and constant external heat resistance between the heating medium and the can surface, (ii) all cans are exactly the same, (iii) the product is conduction-heating. The $F$-values that result from this analysis vary exclusively as a result of the temperature distribution and therefore reflect its potential impact on process variability.

\subsection{Temperature distribution experiments}

The temperature distribution was measured in two industrial scale horizontal water cascading retorts, one with 6 baskets (Retort A) and another with 4 baskets (Retort B). The experiments were performed at the industrial sites, in between normal production batches. Retort A was equipped with 20 calibrated thermocouples (ELLAB type T SSR-60020-G700-SF) distributed in 4 baskets (baskets $1,3,5$ and 6 numbered from the retort end). The probes were located in the centre, top, bottom, left and right surface of the baskets as shown in Fig. 1 and Table 1. The vessel was fully loaded (17 container layers) with pet food in flexible packages.

Thirty calibrated home made thermocouples were placed in basket 2 of Retort B in six layers (see also Fig. 1 and Table 2). In each layer there was a thermocouple in the centre, back, front, left and right surface of the container layer. The data logging time was $15 \mathrm{~s}$ in both experiments.

These two designs are typical of two approaches: analysing the full retort in a single run (case A) and detailing one basket in a retort (case B - in this design the procedure is repeated in all baskets), as detailed in May (1988).

\subsection{Conduction-heating finite element heat transfer model}

In the finite element method (FE), the solution of the set of differential equations and boundary conditions 


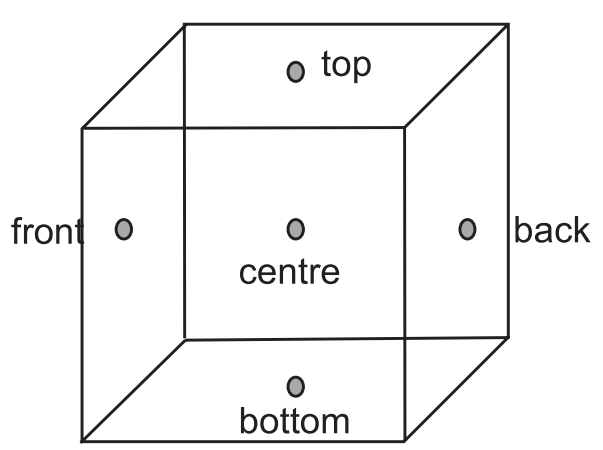

a)

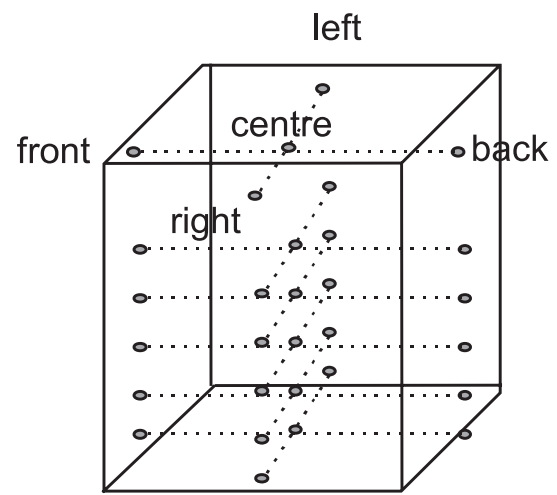

b)

Fig. 1. Thermocouple locations in the experimental temperature distribution trials.

Table 1

Thermocouples and corresponding positions, Retort A

\begin{tabular}{lccccc}
\hline $\begin{array}{l}\text { Thermocouple } \\
\text { number }\end{array}$ & Bottom & Centre & Right & Left & Top \\
\hline Basket 1 & 1 & 2 & 3 & 4 & 5 \\
Basket 3 & 6 & 7 & 8 & 9 & 10 \\
Basket 5 & 11 & 12 & 13 & 14 & 15 \\
Basket 6 & 16 & 17 & 18 & 19 & 20 \\
\hline
\end{tabular}

Table 2

Thermocouples and corresponding positions, Retort B

\begin{tabular}{lcrccc}
\hline $\begin{array}{l}\text { Thermocouple } \\
\text { number }\end{array}$ & Centre & Left & Right & Front & Back \\
\hline Layer 1 & 1 & 2 & 3 & 4 & 5 \\
Layer 2 & 6 & 7 & 8 & 9 & 10 \\
Layer 3 & 11 & 12 & 13 & 14 & 15 \\
Layer 4 & 16 & 17 & 18 & 19 & 20 \\
Layer 5 & 21 & 22 & 23 & 24 & 25 \\
Layer 7 & 26 & 27 & 28 & 29 & 30 \\
\hline
\end{tabular}

established is approximated by a variational statement (Segerlind, 1984):

$\int_{V} W_{i} R \mathrm{~d} v=0$

where $W_{i}$ is a weighing function and $R$ is given by Fourier's second law for a can shaped body, as

$R=\alpha\left(\frac{\partial^{2} T}{\partial z^{2}}+\frac{1}{r} \frac{\partial^{2}(r T)}{\partial r^{2}}\right)-\frac{\partial T}{\partial t} \neq 0$.

Limit conditions:

Initial condition: $\quad t=0, \quad T=T_{0} \quad \forall x, z$,

Symmetry condition: $\quad r=0, \quad \frac{\partial T}{\partial r}=0 \quad \forall t$.
Boundary conditions:
(a) $r=R, \quad-\left.k \frac{\partial T}{\partial r}\right|_{r=R}=h\left(T-T(t)_{\mathrm{R}}\right)$,
(b) $z=0, H, \quad-\left.k \frac{\partial T}{\partial z}\right|_{z=0, H}=h\left(T-T(t)_{\mathrm{R}}\right)$,

where $h$ is the heat transfer coefficient and $T(t)_{\mathrm{R}}$ is the retort temperature as a function of time. In FE the body is subdivided into elements, connected by nodes. Within an element the temperature is calculated by a polynomial (usually low-order) function of $z$ and $r$ for a given time. The derivation of the equations can be found in Segerlind (1984) for Cartesian and axisymmetric co-ordinates and in Misra and Young (1979) for spherical coordinates. After discretisation of the problem domain, it can be shown that the temperature in a given element is

$T^{\mathrm{e}}=\{N\}^{\mathrm{T}}\left\{T_{\mathrm{n}}\right\}$,

where $T_{\mathrm{n}}$ is the nodal temperature vector including the temperatures at the nodes attached to that element and $N^{\mathrm{T}}$ is the row vector of the element shape functions. The solution of Eq. (1) is given by the nodal temperature vector that minimises $R$ in Eq. (2). By substitution of Eq. (3) in Eq. (1) and application of Galerkin's method (stating that $W_{i}=N_{i}$ ) the weighing residual statement can be rewritten as

$[C]\left\{\frac{\partial\{T\}}{\partial t}\right\}+[K]\{T\}+F=0$.

In Eq. (4) the element contributions to the global capacitance matrix $[C]$, to the global conductivity matrix $[K]$ and to the global force vector $\{F\}$ are:

$\left\lfloor c^{(\mathrm{e})}\right\rfloor=\int_{\mathrm{vol}} \rho c_{p} r[N][N]^{\mathrm{T}} \mathrm{d}(\mathrm{vol})$,

$\left\lfloor k^{(\mathrm{e})}\right\rfloor=\int_{\text {vol }}[B][D][B]^{\mathrm{T}} \mathrm{d}($ vol $)+\int_{\text {surf }} h[N][N]^{\mathrm{T}} \mathrm{d}($ surf $)$, 


$$
\left\lfloor f^{(\mathrm{e})}\right\rfloor=\int_{\text {surf }} h T(t)_{\mathrm{R}}[N]^{\mathrm{T}} \mathrm{d}(\operatorname{surf}),
$$

where

$[B]=\left[\begin{array}{l}\frac{\partial[N]}{\partial z} \\ \frac{\partial[N]}{\partial r}\end{array}\right] \quad$ and $\quad[D]=\left[\begin{array}{cc}k_{z z} & 0 \\ 0 & k_{r r}\end{array}\right]$.

Eq. (4) is a system of first-order differential equations which is solved using a finite difference method by transforming the equation into a difference form

$$
\begin{aligned}
([C]+ & \theta \Delta t[K])\{T\}_{t+\Delta t} \\
= & ([C]-(1-\theta) \Delta t[K])\{T\}_{t}+\left((1-\theta)\{F\}_{t}\right. \\
& \left.+\theta\{F\}_{t+\Delta t}\right) .
\end{aligned}
$$

In this work, the unconditionally stable CrankNicholson method was applied $(\theta=1 / 2$, see Chapra \& Canale, 1990). The software package ANSYS5.3 (Houston, USA) was used to build the finite element model and solve the set of equations. The boundary conditions were the discrete values of the corresponding time step, either measured experimentally or predicted by the temperature time-series model.

\subsection{Modelling temperature distribution using time-series}

\subsubsection{Concept}

Retort temperature histories follow a certain pattern with time, involving both systematic and non-systematic (or in other words 'noisy') components. This fact agrees well with the basic concept of time-series models, which involve the analysis of observed data from random processes with time and quantify them in order to predict future values. A general model for an arbitrary variable can be written as

$y_{t}=\hat{y}_{t}+R_{t}$,

where $\hat{y}_{t}$ is the expected value of $y_{t}$ and $R_{t}$ is the residual, with $y_{t}$ representing the variable of interest, in this case the retort temperature. Subscript ' $t$ ' indicates that the continuous variable $y$ is evaluated at specific observation points in time. This fits well with numerical modelling, where the temperature inside the container is also discretised in the space and time domain. Classical timeseries models treat the system as a 'black box' and the factors affecting its behaviour are not studied (Makridakis \& Wheelwright, 1989). These type of models are mostly applied in business sciences for forecasting where it is expected that the value of $y_{t}$ is further divided into trend, cyclic and seasonal components, with some applications in engineering (Morrison, 1984). In the food processing area, Lanoiselle, Candau and Debray (1995) used a similar approach to predict the internal temperature of canned foods during sterilisation. Nicolai and Baerdemaeker (1992) carried out a theoretical study evaluating the effect of the ambient temperature and external heat transfer coefficient in ovens using the Markov process. Zanetti (1992) simulated air pollution dispersion phenomena in 3D using a time-series model.

\subsubsection{Trend model}

In most modern retorts the different phases of the sterilisation cycle can be well defined and it is therefore possible to identify a deterministic behaviour of the heating medium temperature. Therefore, the expected value of $T_{\mathrm{R}}$ is a simple trend, which can be written as simple functions of time. It is worth to note that due to the controlling device some periodical fluctuations may occur, however, this effect can be included in the residual as a cyclic component.

From the recorded temperature data, in Retort A the trend in Eq. (9) was assumed to follow a linear model in the come-up and come-down period of the sterilisation cycle, each with two steps. It was also assumed that after the retort temperature reached the set cooling temperature, it remained constant for the rest of the process. For Retort B, the recorded retort temperatures showed that the come-down phase did not follow a linear relationship, while the come-up trend could be described by a single straight line. In this case, a negative exponential function was selected to model the expected cooling water temperature with time.
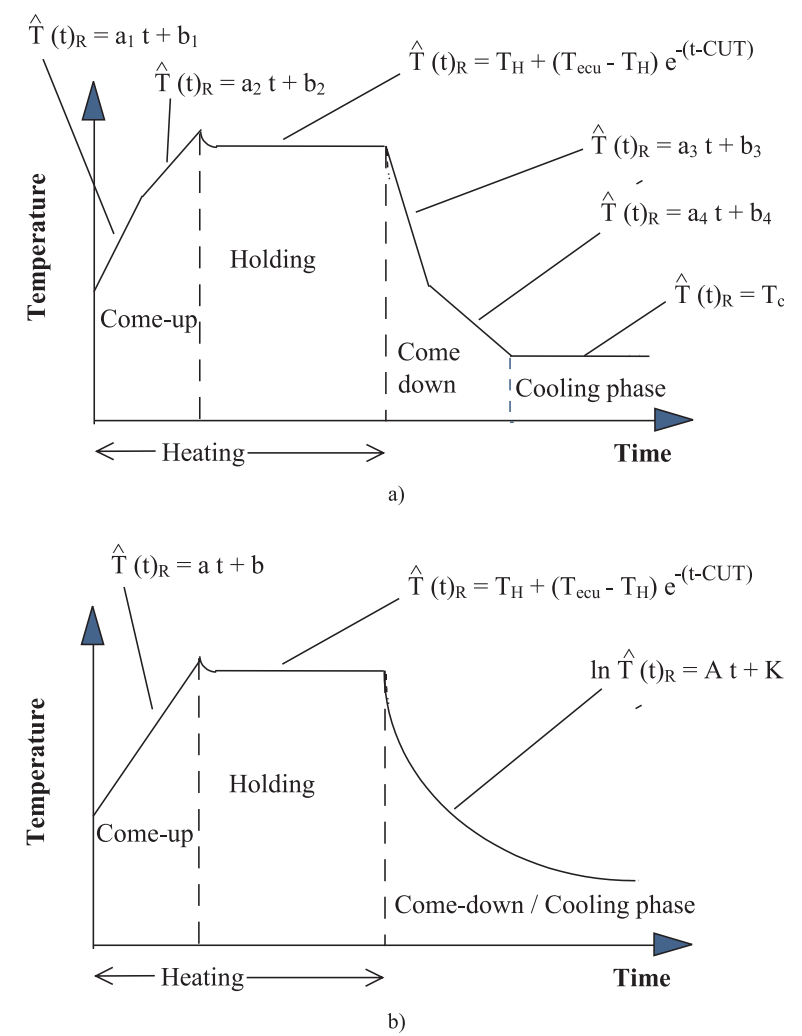

Fig. 2. Mathematical models for the expected retort temperatures (trend functions). 
In order to account for overshooting in the heating phase, the following model was applied to calculate the estimated retort temperature during the holding phase in both retorts

$T(t)_{\mathrm{R}}=T_{\mathrm{H}}+\left(T_{\text {ecu }}-T_{\mathrm{H}}\right) \mathrm{e}^{-(t-\mathrm{CUT})}$.

In Eq. (10), $T_{\mathrm{H}}, T_{\text {ecu }}$ and CUT are the set heating temperature, the temperature at the end of the come-up phase and the come-up time, respectively. The assumptions for the estimated temperature profiles are summarised in Fig. 2.

\subsubsection{Time-series model}

A time-series data set $\left(y_{1}, y_{2}, \ldots, y_{t}\right)$ represents a multivariate system with $R_{1}, R_{2}, \ldots, R_{t}$ showing also a multidimensional distribution, where the elements are not necessarily independent. The residual component in Eq. (9) can be modelled using a general AR$\operatorname{IMA}(p, i, q)(P, I, Q)^{s}$ approach. The term ARIMA refers to a model that includes three components: auto-regression (AR), differencing of the data series (I) and moving averages (MA). A detailed discussion of this type of models can be found in Jarret (1991).

Regressive models in a simple case are generated by finding the relationship $f(x, z, \ldots)$ between the dependent variable (y) and the independent variable(s) $(x, z, \ldots)$ When the dependent variable is constructed from its previous observations, a so-called auto-regressive $\operatorname{AR}(p)$ model is obtained, which can be written as

$y_{t}=f\left(y_{t-1}, y_{t-2}, \ldots, y_{t-p}\right)$.

Not all data series can be characterised by the function presented in Eq. (11). An alternative approach is a MA model that provides a prediction for $y_{t}$ as a linear combination of the forecasting errors $(e)$ in the past

$y_{t}=f\left(e_{t-1}, e_{t-2}, \ldots, e_{t-q}\right)$.

AR and MA models are applicable only for data sets that are said to be stationary or trend free. A very useful method to achieve stationary series is to take the difference of the series by the order of $d$ (I model). Combining the equations above and substituting $y_{t}$ with the residual yields

$$
\begin{aligned}
R_{(t)} & \left(1-B^{1}-\cdots-B^{i}\right)\left(1-\phi_{1} B^{1}-\cdots-\phi_{p} B^{p}\right) \\
& \times\left(1-\phi_{1 s} B^{1 s}-\cdots-\phi_{p s} B^{p s}\right) \\
= & \left(1-\theta_{1} B^{1}-\cdots-\theta_{q} B^{q}\right)\left(1-\theta_{1 s} B^{1 s}-\cdots-\phi_{q s} B^{q s}\right) e_{t},
\end{aligned}
$$

where $B$ is the back shift operator, $B^{n} R_{(t)}=R_{(t-n)}$, and $\phi$ and $\theta$ are the time-series coefficients. Eq. (13) is a general $\operatorname{ARIMA}(p, i, q)(P, I, Q)^{s}$ model including $p+q+P+Q$ different coefficients that must be estimated. A detailed discussion of the fundamentals of multivariate random processes can be found in many textbooks (e.g., Morrison, 1984; Ripley, 1987; Priestley, 1992; Jarret, 1991).
The question arising at this point is how to choose a particular model for a particular system.

\subsubsection{Model selection and estimation of the coefficients}

The most widely used technique to build ARIMA models is the Box-Jenkins method. The advantages of this technique were discussed by Jarret (1991). The selection of $p$ and $q$ requires the evaluation of two sets of statistics, the auto-correlation coefficients and the partial auto-correlation coefficients. Similarly to the correlation coefficient, the auto-correlation coefficient (ACF or $r_{k}$ ) is a measure of the strength of the functional relationship between the dependent and the independent variable(s). However, in this case the independent variable vector is built by the dependent variable with a time lag of $k$ and evaluated as

$$
\begin{aligned}
r_{k}= & \frac{\sum_{t=k}^{n}\left(y_{t}-\bar{y}_{t}\right)\left(y_{t-k}-\bar{y}_{t-k}\right)}{\left[\sum_{t=1}^{n}\left(y_{t}-\bar{y}_{t}\right)^{2}\right]^{1 / 2}\left[\sum_{t=k}^{n}\left(y_{t-k}-\bar{y}_{t-k}\right)^{2}\right]^{1 / 2}}, \\
& -1 \leqslant r_{k} \leqslant 1, \quad k=1,2,3, \ldots, n-1 .
\end{aligned}
$$

The other important parameter is the partial autocorrelation coefficient (PACF). The PACF measures the degree of association between $y_{t}$ and $y_{t-k}$ while keeping the effect of the other time lags on $y$ constant. The calculation of PACF involves the solution of a set of equations that is discussed in Jarret (1991) and Priestley (1992).

The model selection procedure consists of examining the number of significant ACFs and PACFs calculated for a time-history data, at a given level of confidence. $\operatorname{AR}(p)$ model is chosen if the partial auto-correlation coefficients die down exponentially and the ACFs cut-off after $p$ time lag. $\mathrm{A} \operatorname{MA}(q)$ model is selected when the

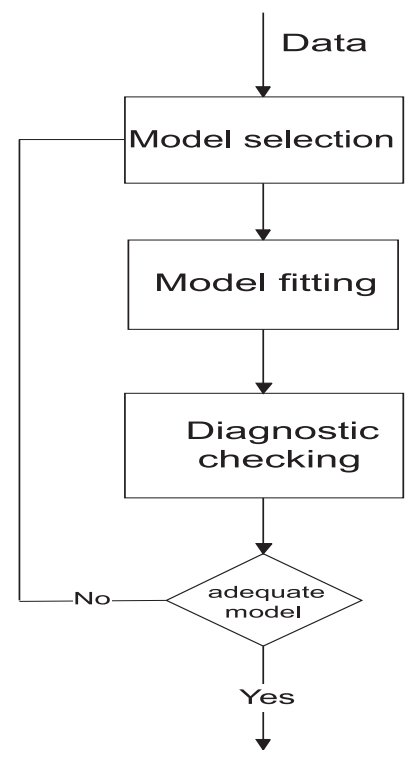

Fig. 3. Schematic representation of the Box-Jenkins method. 
ACFs die down exponentially and there are only $q$ significant PACFs. If both parameters follow an exponential pattern, a combined $\operatorname{ARIMA}(p, i, q)$ model is the most appropriate to describe the data set. In most cases, a model with $p=0,1$ or 2 and $q=0,1$ or 2 is satisfactory.

After the model selection, the Box-Jenkins method proceeds with the parameter estimation by minimising the sum of squares of the errors between the model and the data set. If $q>0$, then the process becomes nonlinear, since $e_{t}$ depends on the previous errors. Methods for performing such iterative processes are provided by most commercial statistical packages.

The last step in building ARIMA models is to check the adequacy of the fitted model using the $\chi^{2}$ statistics for the residual auto-correlation. The model is adequate if the resulting $\chi^{2}$ is less than the value corresponding to a given level of significance. Another way of diagnostic checking is to look for significant PACFs and ACFs in the residual after the model fitting. If no significant values are found, the model is accepted. A schematic visualisation of the Box-Jenkins method is shown in Fig. 3. For the selection and estimation of the proper time-series model, the software STATGRAPHICS (Rockville, USA) was used.

\subsection{Simulation design}

Eq. (8) was solved with the time varying boundary condition using a commercial finite element program package, ANSYS5.3. The powerful user programming capability of the software provided a suitable environment to implement the time-series model within the program package.

The half of a can with $8.6 \mathrm{~cm}$ in diameter and $11.6 \mathrm{~cm}$ in height was modelled and subdivided into 64, 4 node

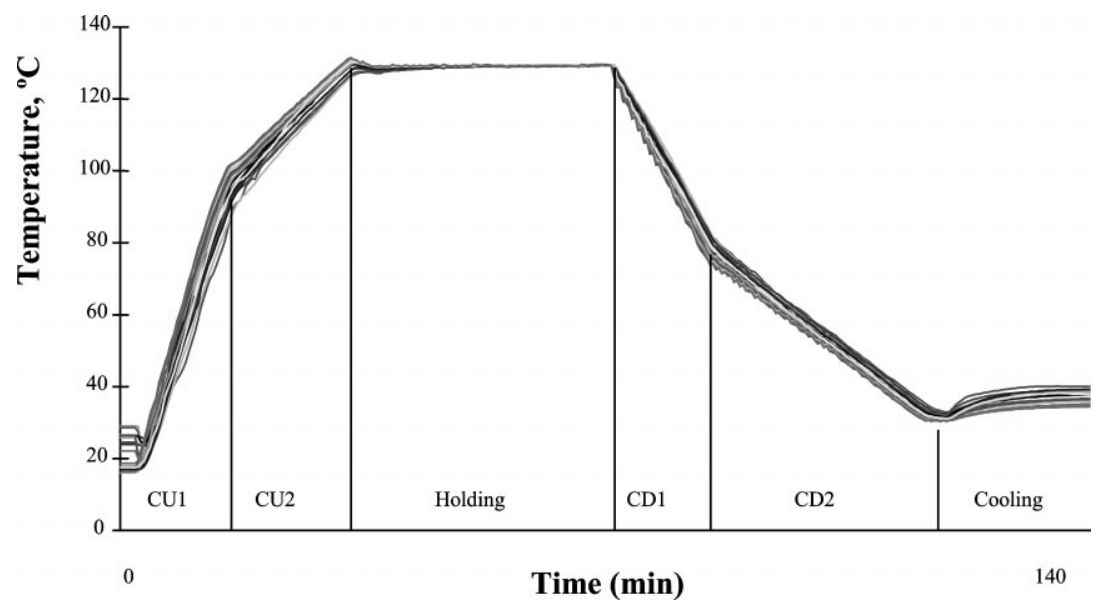

Fig. 4. Experimental time-temperature curves in Run 1 of Retort A.

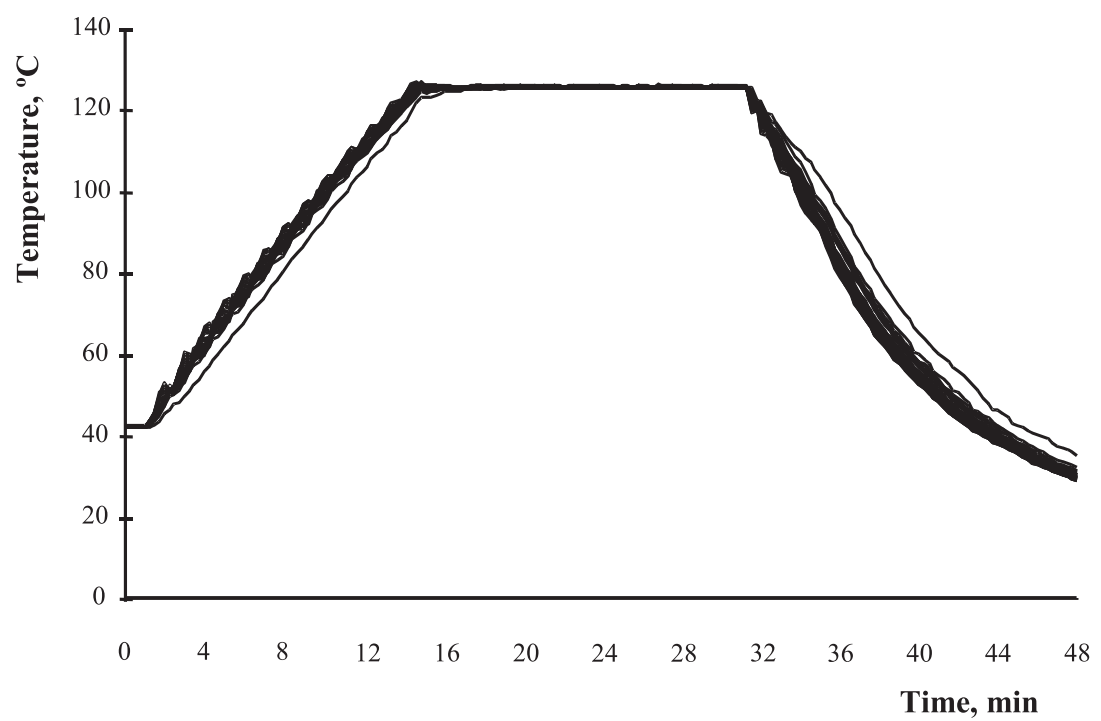

Fig. 5. Experimental time-temperature curves in basket 2 of Retort B. 
quadrilateral axisymmetric elements with decreasing size towards the boundary. The physical properties of the food simulated were $k=1 \mathrm{~W} / \mathrm{m} \mathrm{K}, \rho=1010 \mathrm{~kg} / \mathrm{m}^{3}$ and $c_{p}=3000 \mathrm{~J} / \mathrm{kg}{ }^{\circ} \mathrm{C}$. The initial temperature of the container was $20^{\circ} \mathrm{C}$. The physical properties were selected to be sufficiently high so that at the end of the sterilisation cycle the centre temperature did not affect the lethality value and the effect of the variability of process times was thus eliminated. The heat transfer coefficient over the entire can surface was chosen at a typical value of $500 \mathrm{~W} / \mathrm{m}^{2} \mathrm{~K}$ (Tucker \& Holdsworth, 1991; Tucker, 1991).

The time-temperature data from two retort systems was recorded and used as boundary conditions in the FE conduction-heating model. The parameters of the timeseries model that fitted the whole retort temperature data better were estimated with the Box-Jenkins method. The accuracy of the time-series models in predicting process variability was evaluated by generating 180 temperature histories for Retorts A and B, respectively, with the model, and comparing the resulting $F$-value distributions with the experimental ones.

Normal and independent distributions were used to describe the variability of the parameters of the temperature history model, since there was no statistical evidence for selecting any other distribution. The lethality was calculated using the general method with the temperature data calculated for the geometric centre of the cylindrical container both for the heating phase only, which would be normal industrial practice, and for the total sterilisation cycle including also the effect of the process variability of the cooling phase.

\section{Results and discussion}

Two experimental runs were performed in Retort A. Fig. 4 shows the experimentally recorded retort temperature profiles in the first run, which was selected for subsequent analysis. Four experimental runs were performed in Retort B, each analysing a different basket, and Fig. 5 shows the temperature profiles recorded in basket 2, which was selected for further analysis (being the one where a lowest lethality point was observed). The $F$-values calculated by using the experimental retort temperatures as time varying boundary conditions in the FE model are given in Fig. 6. It can be seen from Fig. 6(a) that for both runs in Retort A the 'coldest point' was located at the bottom centre of basket 1 (see Table 1) considering the whole sterilisation cycle. The corresponding $F$-values for Runs 1 and 2 were 22.77 and $22.86 \mathrm{~min}$, respectively. For the heating phase, there were two least lethality locations in the first batch, one at the bottom centre of basket 1 , as before, but another one at the bottom centre in basket 3 (4.45 and $4.5 \mathrm{~min}$ ). In general, it can be concluded from the results that as expected the $F$-value was higher at the top (thermocouple locations 5, 10, 15

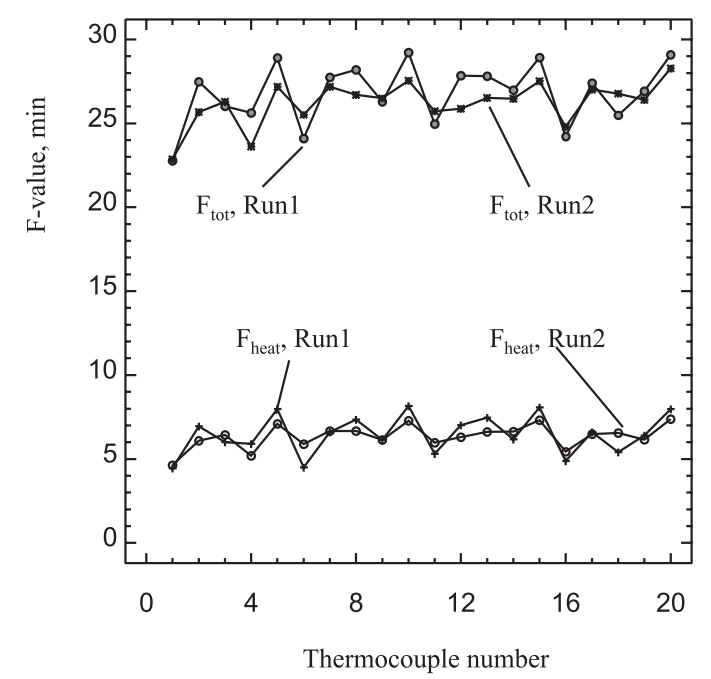

a)

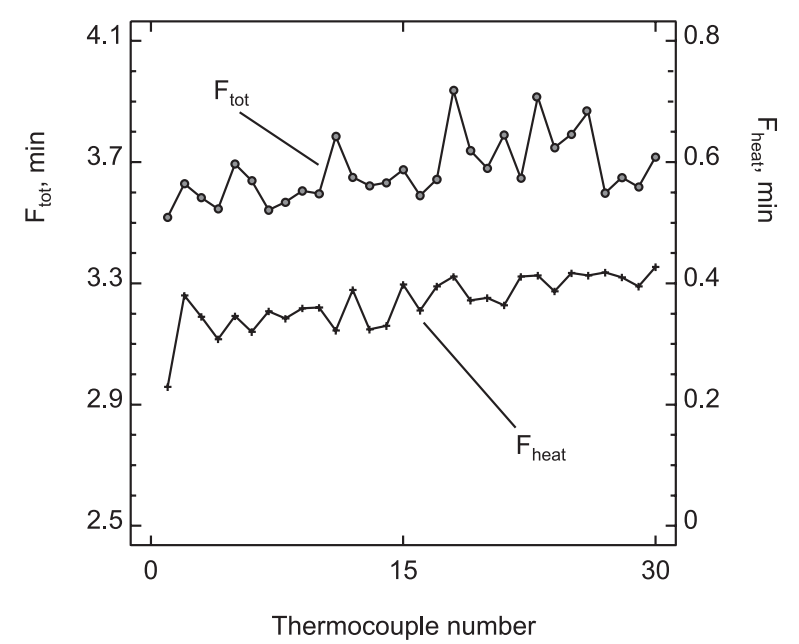

b)

Fig. 6. F-values at the various thermocouple locations in: (a) Retort A; (b) Retort B.

Table 3

Summary statistics of the $F$-value distribution in Retort A

\begin{tabular}{lcccccc}
\hline & Average & $\sigma$ & Min & Max & Range & $\begin{array}{l}\mathrm{CV} \\
(\%)\end{array}$ \\
\hline $\begin{array}{l}\text { Run } 1 \\
F_{\text {heat }} \\
(\text { min) }\end{array}$ & 6.47 & 1.17 & 4.45 & 8.16 & 3.71 & 18.11 \\
$\begin{array}{l}F_{\text {tot }} \\
(\min )\end{array}$ & 26.8 & 1.83 & 22.77 & 29.22 & 6.45 & 6.81 \\
$\begin{array}{l}\text { Run } 2 \\
F_{\text {heat }} \\
(\text { min) }\end{array}$ & 6.35 & 0.7 & 4.64 & 7.38 & 2.74 & 11.11 \\
$\begin{array}{l}F_{\text {tot }} \\
(\min )\end{array}$ & 26.22 & 1.3 & 22.86 & 28.28 & 5.42 & 4.97 \\
\hline
\end{tabular}


and 20) and lower at the bottom of the baskets due to the nature of the water cascading process. Comparison of Runs 1 and 2 indicated that both lethality distributions were statistically similar at $95 \%$ confidence level. Summary statistics of the two $F$-value distributions are shown in Table 3. As the lethality distribu- tions were quite similar in both experimental runs, only the $F$-values from Run 1 were used in the time-series modelling.

Similar results were found for Retort B (see Fig. 8), in which case the least lethality point in basket 2 was located in the bottom layer, while there was a slight in-

Table 4

Parameters of the models of the expected retort temperature

\begin{tabular}{|c|c|c|c|c|}
\hline \multirow[t]{2}{*}{ Parameter } & \multicolumn{2}{|l|}{ Average } & \multicolumn{2}{|l|}{ CV $(\%)$} \\
\hline & Retort A & Retort B & Retort A & Retort B \\
\hline Slope of CU1 $\left(a_{1}\right)\left({ }^{\circ} \mathrm{C} / \mathrm{min}\right)$ & 10.27 & 6.06 & 3.05 & 0.7 \\
\hline Intercept of CU1 $\left(b_{1}\right)\left({ }^{\circ} \mathrm{C}\right)$ & 27.52 & 47.63 & 17.93 & 2.5 \\
\hline Slope of CU2 $\left(a_{2}\right)\left({ }^{\circ} \mathrm{C} / \mathrm{min}\right)$ & 3.35 & - & 7.2 & - \\
\hline Holding temperature $\left(T_{\mathrm{H}}\right)\left({ }^{\circ} \mathrm{C}\right)$ & 129.1 & 125.86 & 0.13 & 0.2 \\
\hline Slope of CD1 $\left(a_{3}\right)\left({ }^{\circ} \mathrm{C} / \mathrm{min}\right)$ & -6.17 & - & 4.89 & - \\
\hline Slope of CD2 $\left(a_{4}\right),{ }^{\circ} \mathrm{C} / \mathrm{min}$ & -2.52 & - & -2.42 & - \\
\hline$A\left({ }^{\circ} \mathrm{C}\right)$ & - & 7.63 & - & 0.4 \\
\hline
\end{tabular}

Table 5

ARIMA models selected and their coefficients

\begin{tabular}{|c|c|c|c|}
\hline & Model & Coefficient & White noise \\
\hline \multicolumn{4}{|l|}{ Retort A } \\
\hline \multirow[t]{2}{*}{ First come-up (CU1) } & $\operatorname{ARIMA}(2,0,0)$ & $\phi_{1}=1.63$ & \\
\hline & & $\phi_{2}=-0.681$ & 0.39 \\
\hline Second come-up (CU2) & $\operatorname{ARIMA}(1,0,0)$ & $\phi_{1}=0.52$ & 0.11 \\
\hline Holding & $\operatorname{ARIMA}(0,0,0)$ & - & 0.15 \\
\hline First come-down (CD1) & $\operatorname{ARIMA}(1,0,0)$ & $\phi_{1}=-0.536$ & 0.19 \\
\hline Second come-down (CD2) & $\operatorname{ARIMA}(1,1,0)$ & $\phi_{1}=-0.47$ & 0.06 \\
\hline Cooling & $\operatorname{ARIMA}(1,1,0)$ & $\phi_{1}=0.648$ & 0.02 \\
\hline \multicolumn{4}{|l|}{ Retort B } \\
\hline \multirow[t]{2}{*}{ Come-up (CU) } & $\operatorname{ARIMA}(1,0,0)(0,0,1)^{4}$ & $\phi_{1}=0.59$ & \\
\hline & & $\theta_{1}=0.81$ & 0.64 \\
\hline Holding & $\operatorname{ARIMA}(0,0,0)$ & - & 0.15 \\
\hline Come-down (CD) & - & - & - \\
\hline
\end{tabular}

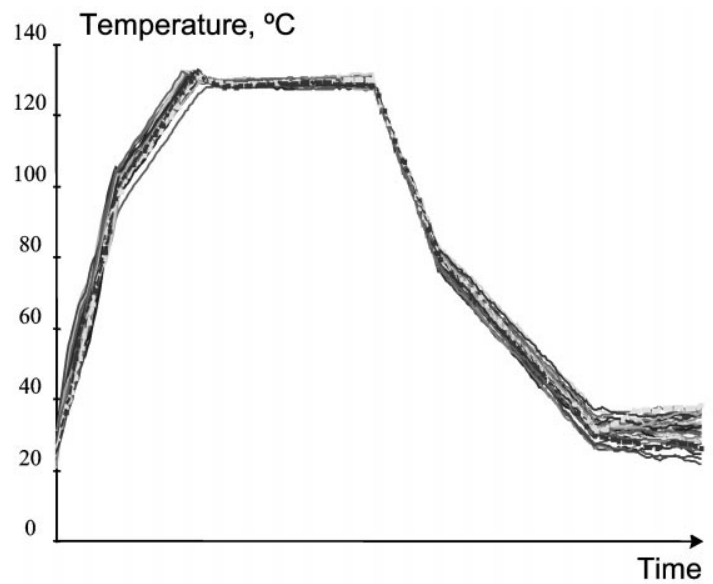

a)

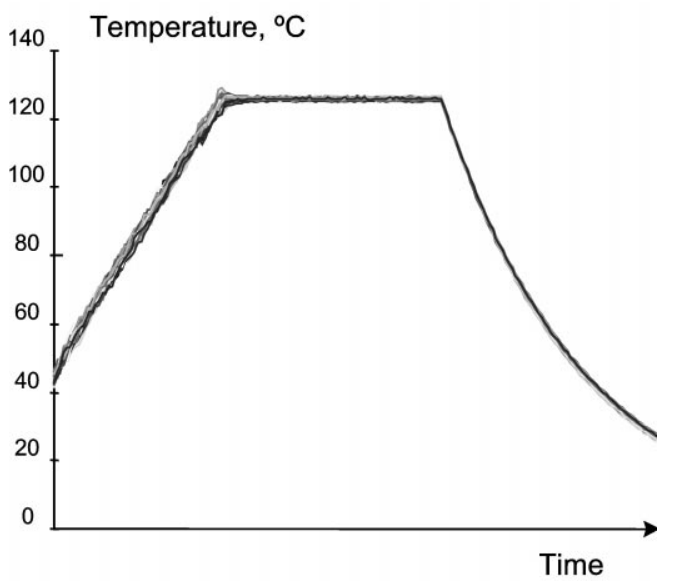

b)

Fig. 7. Some simulated temperature histories for: (a) Retort A; (b) Retort B. 
Table 6

Experimental and simulated $F$-value distribution in Retort A

\begin{tabular}{|c|c|c|c|c|}
\hline \multirow[t]{2}{*}{$F$-value $(\min )$} & \multicolumn{2}{|l|}{$F_{\text {heat }}$} & \multicolumn{2}{|l|}{$F_{\text {tot }}$} \\
\hline & Experimental & Simulated & Experimental & Simulated \\
\hline Count (n) & 20 & 180 & 20 & 180 \\
\hline Average & 6.5 & 7.0 & 26.8 & 27.9 \\
\hline S.D. $(\sigma)$ & 1.2 & 1.33 & 1.8 & 4.1 \\
\hline Minimum & 4.4 & 3.7 & 22.8 & 18.2 \\
\hline Maximum & 8.2 & 10.6 & 29.2 & 35.9 \\
\hline Range & 3.7 & 6.9 & 6.45 & 17.7 \\
\hline CV (\%) & 18.1 & 19.0 & 6.81 & 14.9 \\
\hline
\end{tabular}

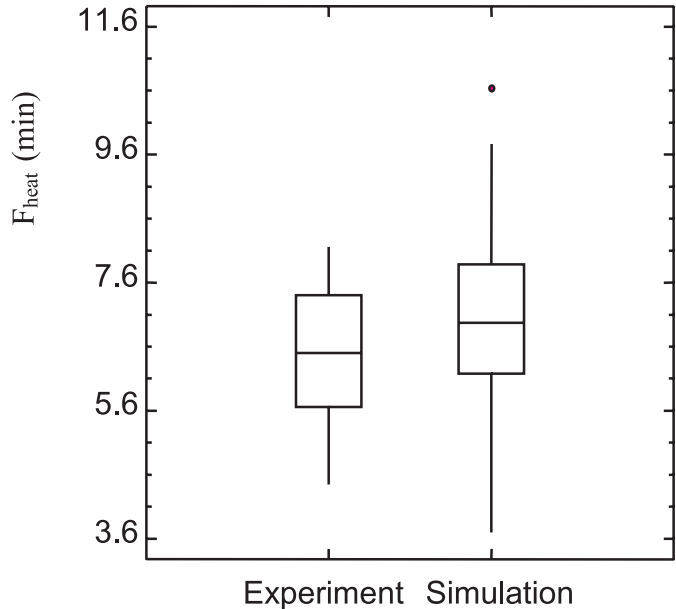

a)

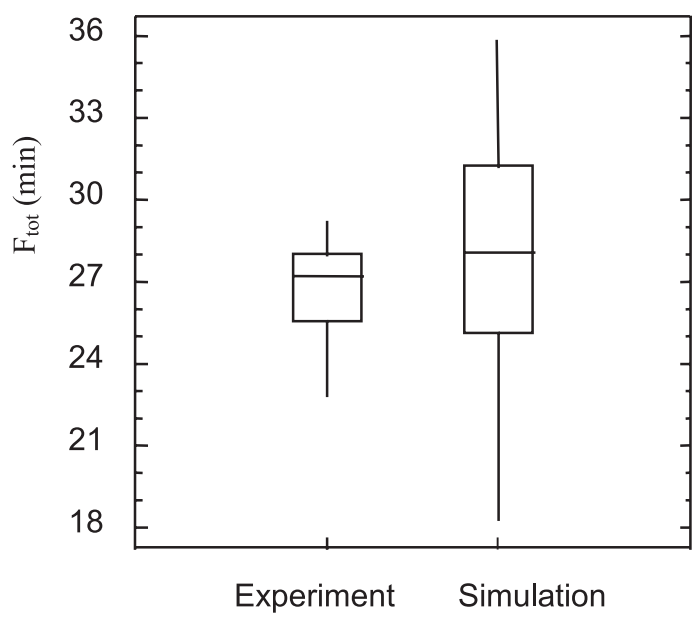

b)

Fig. 8. Experimental and simulated $F$-value distribution in Retort A for: (a) the heating phase (beginning to end of holding); (b) the whole sterilisation cycle.

crease in the $F$-values towards the top layers. Summary statistics of the calculated lethality distribution is given in Table 7. In the top three layers, the least lethality in each layer was found in the centre, but in the bottom three layers this was not so.
For modelling the retort temperature distribution, each profile was divided into a first come-up (CU1), a second come-up (CU2), a holding, a first come-down (CD1), a second come-down (CD2) and a cooling phase in case of Retort A, as shown in Figs. 2(a) and 4(a), and a come-up, a holding and a cooling phase in case of Retort B, Figs. 2(b) and 4(b). The model parameters of the retort trend temperatures are shown in Table 4. The data in this table clearly indicate that Retort A was considerably less uniform than the basket in Retort B monitored, except for the holding temperature, which is logical given the different domains. The factors were quite uniform in the holding and cooling phases, although the initial temperature (intercept of CU1) had a coefficient of variation (CV) as high as $18 \%$ in Retort A. It is noted that some of the factors described in Fig. 2 are not included in Table 4 because they are fixed by those given in order to avoid discontinuities in the simulated temperature profiles. Although there was no strong cross-correlation between the factors, they were not completely independent $\left(R_{\max }^{2}=0.4\right)$. In this work, normally distributed, independent coefficients for the simulation of the expected retort temperature (trends) were considered.

The residuals from the regression analysis were analysed using the Box-Jenkins method. The results for both vessels are shown in Table 5 . The mathematical expressions for the different models can be found in Appendix A. It is noted that although no cyclic behaviour was assumed in the expected temperature models, the residual data indicated a periodical fluctuation in the come-up phase for Retort B which is justified by visual observation of the experimental profiles in Fig. 5. It is also noted that in Retort B there was no time-series model that could describe the residual behaviour in the come-down phase with accuracy. In this case, it was assumed that the retort temperature was equal to its expected value. One hundred and eighty time-temperature profiles were generated according to Tables 4 and 5 . Some of these temperature histories are given in Fig. 7.

Similarly to the experimental temperature profiles, the lethality was calculated for the geometric centre of the food container using the simulated profiles as 
Table 7

Experimental and simulated $F$-value distribution in Retort B

\begin{tabular}{|c|c|c|c|c|}
\hline \multirow[t]{2}{*}{$F$-value (min) } & \multicolumn{2}{|l|}{$F_{\text {heat }}$} & \multicolumn{2}{|l|}{$F_{\text {tot }}$} \\
\hline & Experimental & Simulated & Experimental & Simulated \\
\hline Count $(n)$ & 30 & 160 & 30 & 160 \\
\hline Average & 0.37 & 0.38 & 3.67 & 3.5 \\
\hline S.D. $(\sigma)$ & 0.04 & 0.04 & 0.11 & 0.21 \\
\hline Minimum & 0.23 & 0.3 & 3.52 & 3.04 \\
\hline Maximum & 0.43 & 0.47 & 3.94 & 4.09 \\
\hline Range & 0.2 & 0.17 & 0.42 & 1.05 \\
\hline CV $(\%)$ & 11.71 & 9.04 & 2.91 & 6.02 \\
\hline
\end{tabular}

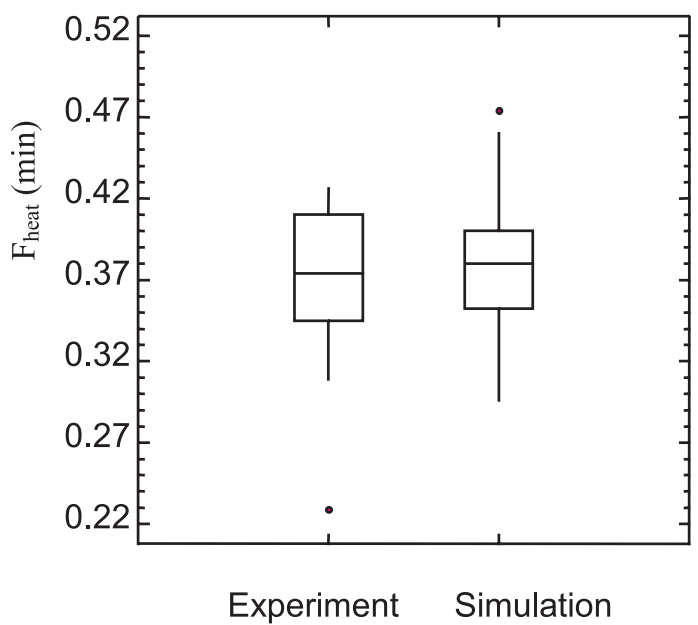

a)

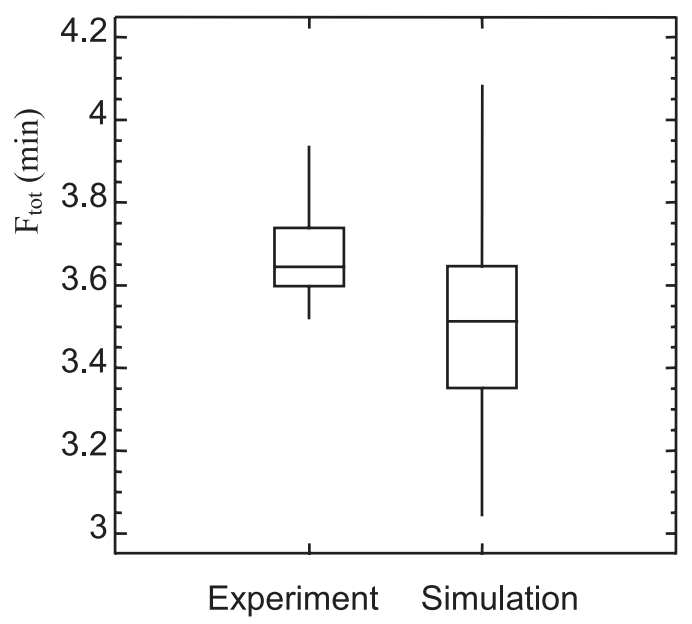

b)

Fig. 9. Experimental and simulated $F$-value distribution in Retort B for: (a) the heating phase (beginning to end of holding); (b) the whole sterilisation cycle.

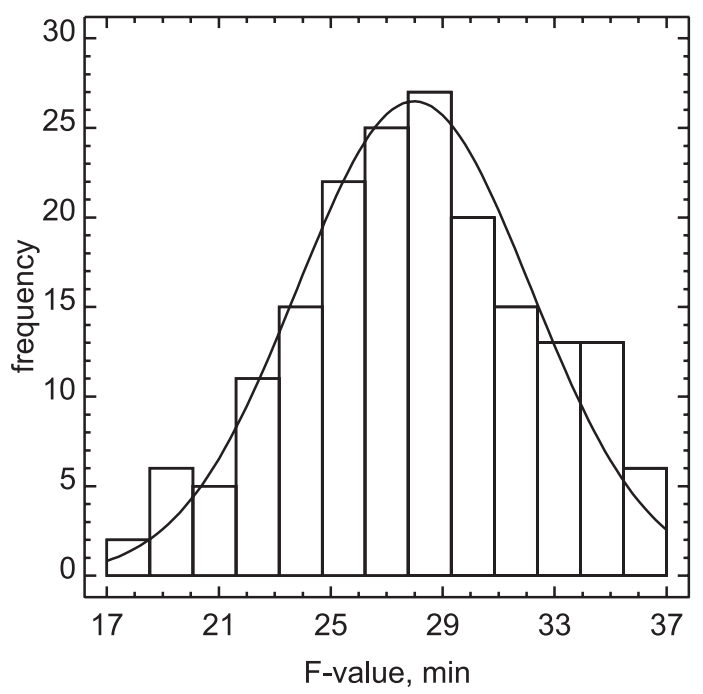

a)

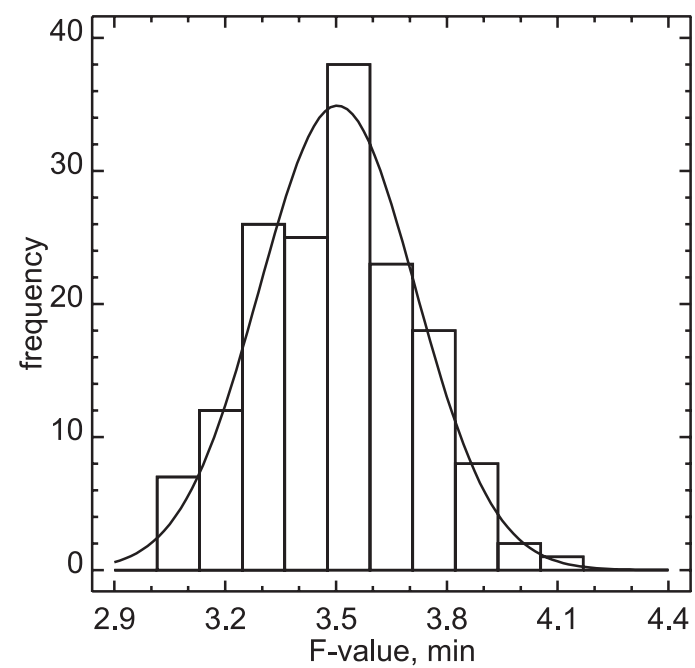

b)

Fig. 10. $F$-value distribution calculated for the whole sterilisation cycle using the time-series model in: (a) Retort A; (b) Retort B. 
boundary condition. The elementary statistics for both the experimental and simulated $F$-value distributions for Retort A are tabulated in Table 6. It can be seen that the simulated temperature distribution resulted in a very similar lethality distribution compared to the experimental one for the heating phase $\left(F_{\text {heat }}\right)$. The difference between the averages were $0.5 \mathrm{~min}$, less than $8 \%$. The simulated 'cold point' was significantly lower than the experimental one, which indicates that this procedure is conservative, as expected. The simulated $F$-value distribution showed a range about two times wider than the experimental one. However, statistical analysis ( $t$-test) indicated no significant difference between the two distributions at 95\% confidence (see Fig. 8(a)). The average $F$-value for the whole sterilisation cycles was also well approximated by the time-series model, with model and experimental values being statistically similar at $95 \%$ confidence level (see Fig. 8(b)). The difference between the experimental and simulated average values was about $4 \%$. However, it is evident that in this case the dispersion of the lethality distribution was much larger. This larger variability might be due to the factors in the time-series model being assumed to have normal distribution, which might not be true. They were also considered to follow independent distributions, but the parameters were slightly correlated. In other words, the locations in the retort which heated faster than the average, cooled faster too, while locations that heated slower, cooled slower. This is also suggested by looking at the experimental standard deviation values in Table 6.

Similar results were found for the basket monitored in Retort B (see Table 7), however in this case the lowest experimental $F$-value was lower than the simulated one. This is probably due to the fact that there was one point in the equipment that resulted in a significantly different temperature profile from the rest of the equipment (see Fig. 5). The probability of having such a profile is very small, therefore this location should be studied in more detail. The experimental range of the lethality distribution excluding this point is $0.31-0.43 \mathrm{~min}$, which is very close to the simulated distribution. Such as before, the simulated retort profiles for the whole cycle resulted in larger lethality variability than the experimental temperature histories. However, statistical analysis of the results indicated that the two $F$-value distributions were similar at 95\% confidence (see Fig. 9).

The number of available simulated lethality data using the time-series model allowed for an evaluation of the type of distribution that the processing values follow. It was found that, based on a $\chi^{2}$ test and the Kolgomorov-Smirnov statistics, the $F$-value approximated well a normal distribution, both for the heating phase and for the whole sterilisation cycle. Histograms for $F_{\text {tot }}$ are given in Fig. 10. Similar distributions were obtained for the sterilisation values calculated for the heating phase alone. The 95\% confidence limits calcu- lated based on the fitted distributions were 4.98-9.6 and 19.1-36.16 $\mathrm{min}$ for $F_{\text {heat }}$ and $F_{\text {tot }}$, respectively, in Retort A. Significantly smaller intervals were obtained for the corresponding 95\% confidence limits, $0.31-0.45$ and 3.09-3.92 min, in Retort B, where only a single basket was monitored.

\section{Conclusions}

The temperature distribution in two different retort systems was analysed and modelled using a finite element conduction-heating model. The temperature distribution impact was evaluated in terms of the resulting lethality distribution at the geometric centre of the container. The expected retort temperature (trend) was modelled by simple functions of time. The time variability was approximated by ARIMA time-series models. The form of the time-series was selected and the parameters were obtained from experimental data using the Box-Jenkins method.

The experimental and simulated lethality distributions compared well, showing that the basic assumption that residuals after application of ARIMA models were normally distributed is acceptable. It was found that the 'generated' $F$-value distribution was very similar to the experimental one for the heating/holding phase, implying that the model approach would recreate correctly the impact of variability on the lethality distribution that can be expected in the retort cycle. Considering the total process, the average lethality was accurately approximated by the simulation, however the standard deviation was smaller for the experimental values. The reason for the larger simulated lethality distribution might be that the factors in the model were not completely independent and the assumption of normal distribution may also be challenged.

The advantage of using the time-series is that a large number of retort temperature histories can be generated in a short time while the information gathered from temperature distribution experiments are highly limited by the number of available thermocouples and the executable experimental runs due to cost reasons. The timeseries models allowed for the evaluation of the $F$-value distribution due to the variability in the heating medium temperature. The results indicated a normal distribution for both equipments.

\section{Acknowledgements}

The authors would like to acknowledge the AIR2CT94-1017 European project (AIR programme) for financial support. The first author would also like to acknowledge financial support from Fundação para a 
Ciência e Tecnologia within the framework of the PRAXIS XXI program.

\section{Appendix A}

$\begin{array}{ll}\operatorname{ARIMA}(0,0,0) & R_{t}=e_{t}\left(0, \sigma^{2}\right) \\ \operatorname{ARIMA}(1,0,0) & R_{t}=\phi_{1} R_{t-1}+e_{t}\left(0, \sigma^{2}\right) \\ \operatorname{ARIMA}(2,0,0) & R_{t}=\phi_{1} R_{t-1}+\phi_{2} R_{t-2}+e_{t}\left(0, \sigma^{2}\right) \\ \operatorname{ARIMA}(1,1,0) & R_{t}=R_{t-1}+\phi 1\left(R_{t-1}-R_{t-2}\right)+e_{t}\left(0, \sigma^{2}\right) \\ \operatorname{ARIMA}(1,0,0)(0,0,1)^{4} & R_{t}=\phi_{1} R_{t-1}-\theta_{t-4} e_{t-4}\left(0, \delta^{2}\right)+e_{t}\left(0, \sigma^{2}\right)\end{array}$

\section{References}

Adams, H. W., \& Hardt-English, P. K. (1990). Determining temperature distribution in cascading water retorts. Food Technology, 12, 110-112.

Chapra, C. C., \& Canale, R. P. (1990). Numerical methods for engineers. Singapore: McGraw-Hill.

Campbell, S., \& Ramaswamy, H. S. (1992). Distribution of heat transfer rate and lethality in a single basket water cascade retort Journal of Food Process Engineering, 15, 31-48.

Hayakawa, K. I., Massaguer, P., \& Trout, R. J. (1988). Statistical variability of thermal process lethality in conduction heating foodcomputerized simulation. Journal of Food Science, 53, 1887-1893.

Hicks, E. W. (1961). Uncertainties in canning process calculations. Journal of Food Science, 26, 218-226.

Jarret, J. (1991). Business forecasting methods. Brasilia: Blackwell.

Lanoiselle, J. L., Candau, Y., \& Debray, E. (1995). Predicting internal temperature of canned foods during thermal processing using a linear recursive model. Journal of Food Science, 60, 833-835.

Lund, D. B. (1978). Statistical analysis of thermal process calculations. Food Technology, 32, 76-78.
May, N. 1988. Guidelines for assessment of overpressure rotary retorts, Project Document AIR2-CT94-1017. Chipping Campden, UK: Campden \& Chorleywood Food Research Association.

Makridakis, S., \& Wheelwright, S.C. (1989). Forecasting methods for management (pp. 49-220). Singapore: Wiley.

Misra, R. N., \& Young, J. H. (1979). The finite element approach for solution of transient heat transfer in a sphere. Transactions of the ASAE, 22, 944-949.

Morrison, D. F. (1984). Multivariate statistical methods. Auckland: McGraw-Hill.

Nicolai, B. M., \& Baerdemaeker, J. (1992). Simulation of heat transfer in foods with stochastic initial and boundary conditions. Food and Bioproducts Processing, 70, 78-82.

Nicolai, B. (1994). Modelling and uncertainty propagation analysis of thermal food processes. Ph.D. thesis. Agricultural Engineering Department, Katholieke Universiteit te Leuven, Leuven, Belgium.

Park, D. J., Cables, L. J., \& Collins, K. M. (1990). Determining temperature distribution in rotary full-immersion hot-water sterilizers. Food Technology, 44, 113-118.

Patino, H., \& Heil, J. R. (1985). A statistical approach to error analysis in thermal process calculations. Journal of Food Science, 50, 1110 1114.

Priestley, M. B. (1992). Spectral analysis and time series. London: Academic Press.

Ripley, B. D. (1987). Stochastic simulation. New York: Wiley.

Segerlind, L. J. (1984). Applied finite element analysis. New York: Wiley.

Tucker, G. S. (1991). Development and use of numerical techniques for improved thermal process calculations and control. Food Control, 2, 15-19.

Tucker, G. S., \& Holdsworth, S. D. (1991). Mathematical modelling of sterilisation and cooking processes for heat preserved foods. Food and Bioproducts Processing, 69, 5-12.

Tung, M. A., Britt, I. J., \& Ramaswamy, H. S. (1990). Food sterilization in steam/air retorts. Food Technology, 44, 105-109.

Zanetti, P. (1992). Particle modeling and its application for simulating air pollution phenomena. In P. Melli, \& P. Zanetti, Enviromental modeling (pp. 211-241). London: Elsevier (Chapter 11). 